\title{
Epidemiology And Antifungal Susceptibility Patterns Of Invasive Fungal Infections From 2012 To 20 I4 In A Teaching Hospital In Central China
}

This article was published in the following Dove Press journal: Infection and Drug Resistance

\begin{abstract}
Hui Xu, ${ }^{1} *$ Shu-Ying Yu, ${ }^{2-4}, *$ MengLan Zhou, (iD) ${ }^{2-4}$ Ya-Ting Ning, (D) ${ }^{2-4}$ Meng Xiao, ${ }^{2,4}$ Xiao-Gai Li,' Meng Chen, ${ }^{2,5}$ Fanrong Kong, ${ }^{6}$ Sharon Chen, ${ }^{6}$ Liang Ming,' Ying-Chun $\mathrm{Xu}^{2,3}$

'Department of Clinical Laboratory, The First Affiliated Hospital of Zhengzhou University and Key Laboratory of Clinical Laboratory of Henan Province, Henan 450003, People's Republic of China;

${ }^{2}$ Department of Clinical Laboratory, Peking Union Medical College Hospital, Chinese

Academy of Medical Sciences, Beijing 100730, People's Republic of China; ${ }^{3}$ Graduate School, Peking Union Medical College, Chinese Academy of Medical Sciences, Beijing 100730, People's Republic of China; ${ }^{4}$ Beijing Key Laboratory for Mechanisms Research and Precision Diagnosis of Invasive Fungal Diseases (BZ0447), Beijing, People's Republic of China; ${ }^{5}$ Department of Clinical Laboratory, Beijing Pinggu Hospital of Traditional Chinese Medicine, Beijing 101200, People's Republic of China; ${ }^{6} \mathrm{Centre}$ for Infectious Diseases and Microbiology Laboratory Services, ICPMR - New South Wales Health Pathology, The University of Sydney, Westmead Hospital, Westmead, NSW, Australia
\end{abstract}

*These authors contributed equally to this work

Correspondence: Liang Ming Department of Clinical Laboratory, The First Affiliated Hospital of Zhengzhou University, IE Jianshe Road, Zhengzhou, Henan 450052, People's Republic of China

Tel +86 37l 669l 3l I8

Email mingliang3072@163.com

Ying-Chun $\mathrm{Xu}$

Department of Clinical Laboratory, Peking Union Medical College Hospital, Chinese

Academy of Medical Sciences, No.I

Shuaifuyuan, Wangfujing, Dongcheng

District, Beijing 100730, People's Republic of

China

$\mathrm{Tel} / \mathrm{Fax}+861069159766$

Email xycpumch@139.com
Introduction: As participants of the national China Hospital Invasive Fungal Surveillance Net program, we sought to describe the epidemiology and antifungal susceptibility patterns of yeast isolates obtained from patients with invasive fungal infection at the First Affiliated Hospital of Zhengzhou University, China.

Methods: A total of 434 yeast isolates recovered from blood and other sterile body fluids were identified to species by matrix-assisted laser desorption ionization -time of flight mass spectrometry with or without supplementation by DNA sequencing. Antifungal susceptibilities were determined by Sensititre YeastOne ${ }^{\mathrm{TM}}$ YO10 methodology.

Results: Candida albicans was the most common causative species (33.9\% of isolates) but significantly decreased in frequency from $37.2 \%$ to $27.7 \%$ from 2012 to 2014 . C. tropicalis was the next most common pathogen (25.1\%), followed by $C$. parapsilosis complex $(17.3 \%)$, C. glabrata $(9 \%)$, and C. pelliculosa $(6.7 \%)$, with other species comprising $8 \%$ of isolates. Caspofungin, micafungin, and anidulafungin exhibited potent in vitro activities against the majority of Candida isolates. Azoles demonstrated in vitro activities against $C$. albicans with a susceptibility rate of $>95 \%$ and against $C$. parapsilosis complex, $>95 \%$ isolates were susceptible. Among C. tropicalis and C. glabrata isolates, resistance rates to fluconazole and voriconazole were $11.9 \%, 9.1 \%$ and $7.7 \%, 28.2 \%$, respectively. Of note, C. pelliculosa had a high incidence rate in newborns and high rates of resistance to fluconazole and voriconazole of $55.2 \%$ and $41.4 \%$, respectively.

Conclusion: The present study provided valuable local surveillance data on the epidemiology and antifungal susceptibilities of invasive yeast species, which is essential for guiding antifungal treatment protocol development.

Keywords: invasive yeast infection, epidemiology, antifungal susceptibility

\section{Introduction}

Invasive fungal infections (IFIs) are major global health threat, particularly the immunocompromised and critically ill, and are widely recognized as a major cause of substantial morbidity and mortality and excess hospital costs. ${ }^{1,2}$ Although Candida albicans remains the most predominant species responsible for invasive infections, its dominance has decreased as non-C. albicans yeast species are increasingly encountered. ${ }^{3,4}$ It is widely appreciated that non- $C$. albicans yeast species are often less susceptible to antifungal drugs than $C$. albicans which impact on clinical outcomes. 5,6

Epidemiology data including species distribution and antifungal susceptibility profile of the causative yeast species are essential to improve the overall outcomes. 5,7 
However, species distribution varies considerably with geographic and institution or hospital, and even within any one institution. ${ }^{8,9}$ Targeted antifungal therapy or antifungal prophylaxis regimens are contingent on the timely diagnosis and antifungal susceptibility testing. ${ }^{8,10}$ Henan is the third largest province in China with a population of about 96 million, and knowledge of species distribution and their antifungal susceptibility profile of invasive yeast infections in this populous region will have substantial clinical impact.

The national China Hospital Invasive Fungal Surveillance Net (CHIF-NET) program across China has provided updated information on epidemiological data for IFIs. $^{3,5,11}$ As a participant of this national surveillance program, the present study sought to describe the epidemiology and antifungal susceptibility patterns of yeast isolates obtained from IFI patients who presented to the First Affiliated Hospital of Zhengzhou University in Zhengzhou, China.

\section{Materials And Methods Study Design And Ethics Statement}

Yeast isolates were collected consecutively over the 3-year study period (August 2012 to July 2014) from patients admitted to the First Affiliated Hospital of Zhengzhou University enrolled in the CHIF-NET study. The First Affiliated Hospital of Zhengzhou University is an 8000bed major tertiary teaching hospital in central China with a range of specialty services. Non-duplicated isolates recovered from blood or other sterile body fluids were included according to the criteria described previously. ${ }^{11}$ The study was approved by the Human Research Ethics Committee of Peking Union Medical College Hospital (No. S-263).

\section{Species Identification}

Yeast isolates were initially identified at the First Affiliated Hospital of Zhengzhou University using chromogenic agar and the VITEK $2 \circledR$ compact system (bioMérieux, Marcy 1'Etoile, France). Accurate identification was confirmed to species level at a central laboratory (Peking Union Medical College Hospital) by matrix-assisted laser desorption ionization-time of flight mass spectrometry (MALDI-TOF MS) using the VITEK MS system (v2.0, IVD database, bioMérieux) supplemented with internal transcribed spacer (ITS) sequencing as previously described. ${ }^{12}$ DNA extraction and amplification of the ITS region was carried out with primer pairs ITS1/ITS4 as described by Zhang et al. ${ }^{12}$ The PCR products were sequenced in both directions using corresponding PCR amplification primer pairs at Ruibiotech Co. Ltd. (Beijing, China) using the DNA analyzer ABI 3730XL system (Applied Biosystems, Foster City, CA). Species identification was performed by comparing the obtained sequences against GenBank database with nucleotide Basic Local Alignment Search Tool (BLASTn, http:// blast.ncbi.nlm.nih.gov).

\section{Antifungal Susceptibility Testing}

The in vitro susceptibility to nine antifungal drugs (fluconazole, voriconazole, itraconazole, posaconazole, caspofungin, micafungin, anidulafungin, amphotericin $\mathrm{B}$, and 5-flucytosine) was determined by Sensititre YeastOne ${ }^{\mathrm{TM}}$ YO10 methodology (Thermo Scientific, USA) following the manufacturer's instructions. Minimum inhibitory concentration (MIC) values for yeast isolates were determined by the Sensititre YeastOne YO10 method and interpreted by species-specific clinical breakpoints (CBPs) as recommended by the CLSI M60 method. ${ }^{13}$ With regard to species for which there are no CBPs we used epidemiological cutoff values (ECVs) to differentiate wild-type (WT) from non-WT isolates according to CLSI M59 method ${ }^{14}$ and ECVs from the study by Pfaller et al. ${ }^{15}$ C. parapsilosis ATCC 22019 and C. krusei ATCC 6258 were the quality control strains.

\section{Statistical Analysis}

All statistical analyses were performed using IBM SPSS software (version 24.0; IBM SPSS Inc., New York, USA). Categorical variables were compared using $\chi^{2}$ or Fisher's exact test. A $P$ value of 0.05 was significant.

\section{Results}

\section{Patient Characteristics And Species Distribution}

Table 1 summarizes the patient characteristics and species distribution. Overall, a total of 434 non-duplicated yeast isolates from separate patients were collected; there were more isolates from males than females ( 57.4 vs $42.6 \%$, respectively). Isolates were cultured from 434 patients aged 0 to 96 years (average age 50.8 years). Invasive infection occurred mainly in the age groups 15-49 and over 65 years, accounting for 32\% (139/434) and 30.9\% (134/434), respectively. For infants (aged 0-1 year), $C$. pelliculosa was a predominant pathogen with an isolation rate the same as that of C. albicans (both 35.5\%) (see species distribution below). The majority of the isolates 
Table I Clinical Characteristics Of Patients And Species Distribution Of The Yeast Isolates

\begin{tabular}{|c|c|c|c|c|c|c|c|c|}
\hline \multirow{2}{*}{$\begin{array}{l}\text { Patients } \\
\text { Characteristic }\end{array}$} & \multicolumn{8}{|c|}{ Number (\%) Of Isolates } \\
\hline & Total & $\begin{array}{l}\text { Candida } \\
\text { Albicans }\end{array}$ & $\begin{array}{l}\text { Candida } \\
\text { Tropicalis }\end{array}$ & $\begin{array}{l}\text { Candida } \\
\text { Parapsilosis } \\
\text { Complex }^{\mathrm{a}}\end{array}$ & $\begin{array}{l}\text { Candida } \\
\text { Glabrata }\end{array}$ & $\begin{array}{l}\text { Candida } \\
\text { Pelliculosa }\end{array}$ & $\begin{array}{l}\text { Cryptococcus } \\
\text { Neoformans }\end{array}$ & Others $^{b}$ \\
\hline \multicolumn{9}{|l|}{ Gender } \\
\hline Female & $185(42.6)$ & $63(34.1)$ & $40(21.6)$ & $26(14.1)$ & $24(13.0)$ & $13(7.0)$ & $6(3.2)$ & $13(7.0)$ \\
\hline Male & $249(57.4)$ & $84(33.7)$ & $69(27.7)$ & 49 (19.7) & $15(6.0)$ & $16(6.4)$ & $5(2.0)$ & II (4.4) \\
\hline \multicolumn{9}{|l|}{ Age (years) } \\
\hline $0-1$ & $31(7.1)$ & II (35.5) & $5(16.1)$ & I (3.2) & $3(9.7)$ & II (35.5) & - & - \\
\hline $2-14$ & $15(3.5)$ & $3(20.0)$ & $7(46.7)$ & - & - & I (6.7) & $4(26.6)$ & - \\
\hline $15-49$ & $139(32.0)$ & $41(29.5)$ & $38(27.4)$ & $33(23.7)$ & $10(7.2)$ & $5(3.6)$ & $2(1.4)$ & $10(7.2)$ \\
\hline $50-65$ & II5 (26.5) & $43(37.4)$ & $28(24.3)$ & $14(12.2)$ & II (9.6) & $6(5.2)$ & $5(4.3)$ & $8(7.0)$ \\
\hline$>65$ & $134(30.9)$ & $49(36.6)$ & $31(23.1)$ & $27(20.1)$ & $15(11.2)$ & $6(4.5)$ & - & $6(4.5)$ \\
\hline \multicolumn{9}{|l|}{ Ward Type } \\
\hline ICU & $168(38.7)$ & $69(4 I . I)$ & $43(25.6)$ & $28(16.6)$ & $15(8.9)$ & $4(2.4)$ & $4(2.4)$ & $5(3.0)$ \\
\hline Surgical department & $152(35.0)$ & $56(36.8)$ & $39(25.7)$ & $25(16.4)$ & $13(8.6)$ & $10(6.6)$ & - & $9(5.9)$ \\
\hline Medical department & $5 \mathrm{I}(1 \mathrm{I} .8)$ & $(7 \mid 3.7)$ & $12(23.5)$ & $12(23.5)$ & $5(9.8)$ & $3(5.9)$ & $6(11.8)$ & $6(11.8)$ \\
\hline Others & $63(14.5)$ & $15(23.8)$ & $15(23.8)$ & $10(15.9)$ & $6(9.5)$ & $12(19.0)$ & I (I.6) & $4(6.4)$ \\
\hline \multicolumn{9}{|l|}{ Separated year } \\
\hline 2012 & $129(29.7)$ & $48(37.2)$ & $34(26.4)$ & $31(24.0)$ & $6(4.7)$ & $3(2.3)$ & I (0.7) & $6(4.7)$ \\
\hline 2013 & II4 (26.3) & $46(40.3)$ & $17(14.9)$ & $19(16.7)$ & 17 (14.9) & $6(5.3)$ & $4(3.5)$ & $5(4.4)$ \\
\hline 2014 & $191(44.0)$ & $53(27.7)$ & $58(30.4)$ & $25(13.1)$ & $16(8.4)$ & $20(10.5)$ & $6(3.1)$ & $13(6.8)$ \\
\hline \multicolumn{9}{|l|}{ Separated sites } \\
\hline Blood & $224(5 \mid .6)$ & $72(32.1)$ & $52(23.2)$ & $36(16.1)$ & $27(12.1)$ & $22(9.8)$ & $3(1.3)$ & $12(5.4)$ \\
\hline CVC & $66(15.2)$ & $17(25.8)$ & $17(25.8)$ & $20(30.3)$ & I (I.4) & $6(9.1)$ & - & $5(7.6)$ \\
\hline pus & $58(13.4)$ & $22(37.9)$ & $16(27.6)$ & II (19.0) & $5(8.6)$ & - & - & $4(6.9)$ \\
\hline Ascitic fluid & $38(8.8)$ & $19(50.0)$ & $10(26.3)$ & $3(7.9)$ & $4(10.5)$ & - & - & $2(5.3)$ \\
\hline Pleural fluid & $13(3.0)$ & $8(6 \mid .5)$ & $2(15.4)$ & I (7.7) & - & - & $2(15.4)$ & - \\
\hline CSF & $12(2.7)$ & $3(25.0)$ & $4(33.3)$ & I (8.4) & - & - & $4(33.3)$ & - \\
\hline Bile & $7(1.6)$ & $2(28.6)$ & $4(57.1)$ & - & I (I4.3) & - & - & - \\
\hline Joint fluid & $6(1.4)$ & I (I6.6) & I (I6.6) & $2(33.6)$ & I (I6.6) & - & - & I (I6.6) \\
\hline Tissue & $6(1.4)$ & $2(33.3)$ & $2(33.3)$ & - & - & I (I6.7) & I (I6.7) & - \\
\hline Others & $4(0.9)$ & I (20.0) & I (20.0) & I (20.0) & - & - & I (20.0) & - \\
\hline Total & 434 & $\begin{array}{l}147 \\
(33.9)\end{array}$ & $109(25.1)$ & 75 (17.3) & $39(9.0)$ & $29(6.7)$ & II (2.5) & $24(5.5)$ \\
\hline
\end{tabular}

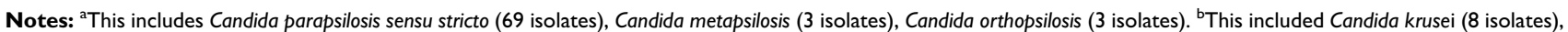
Candida guilliermondii (4 isolates), Candida haemulonii (4 isolates), Candida lusitaniae (I isolate), Candida intermedia (I isolate), Kodamaea ohmeri (3 isolates), Rhodotorula mucilaginosa (2 isolates) and Trichosporon asahii ( 1 isolate).

were from patients in the intensive care unit (ICU) (168, $38.7 \%$ ), surgical departments $(152,35 \%)$, and medical departments $(51,11.8 \%)$.

Overall, 19 species of yeast were identified among the 434 isolates of which $C$. albicans was the most common species (147 isolates, 33.9\%). Non-albicans Candida species accounted for 270 isolates $(62.2 \%)$ - among these, $C$. tropicalis was the most frequent species $(25.1 \%, 109$ isolates). C. parapsilosis complex was the third common species (75 isolates, 17.3\%), which consisted of C. parapsilosis sensu stricto (69/75 isolates, 15.9\%), C. metapsilosis $(3 / 75$ isolates, $0.7 \%$ ), and $C$. orthopsilosis (3/75 isolates, $0.7 \%)$, followed by the C. glabrata (39 isolates, 9\%) and C. pelliculosa (29 isolates, 6.7\%). In addition, there were small 
numbers of isolates of other Candida species including $C$. krusei (8 isolates), C. guilliermondii (4 isolates), C. haemulonii (4 isolates), and each one isolate of $C$. intermedia and C. lusitaniae. Cryptococcus neoformans (11 isolates, 2.5\%) was the most common non-Candida yeast species, followed by Kodamaea ohmeri (3 isolates), Rhodotorula mucilaginosa (2 isolates) and Trichosporon asahii (1 isolate) (Table 1).

Over the 3-year study period, the frequency of all yeast isolates decreased from $29.7 \%$ to $26.3 \%$ then markedly increased to $44 \%$ from 2012 to $2014(P<0.001)$, with the frequency of non-Candida species also moderately increasing from $3.1 \%$ to $4.7 \%(P<0.5)$. With regard to individual species, the isolation rate of $C$. albicans decreased significantly from $37.2 \%$ to $27.7 \%(P=0.095)$, whilst the isolation rate of non-albicans Candida reached up to $29.7 \%$ in 2014 (compared with $17.7 \%$ in 2012, $P<0.001)$. Of these, the proportion of $C$. tropicalis of $30.4 \%$ collected from 2014 exceeded the frequency of $26.4 \%$ and $14.9 \%$ collected during 2012 and 2013, respectively $(P=0.5)$. The isolation rate of $C$. glabrata was $14.9 \%$ and $8.4 \%$ of 2013 and 2014, respectively, higher than that in $2012(4.7 \%, P<0.5)$. Of note, the isolation rate of $C$. pelliculosa rose from $2.3 \%$ to $10.5 \%$ during the 3 years $(P<0.05)$. Conversely, the frequency of $C$. parapsilosis complex declined from $24 \%$ to $13.1 \%$ from 2012 to 2014 $(P<0.05)$.

Table 1 also shows species distribution of the 434 yeast isolates according to the source of specimens. The most common specimen type was blood $(51.6 \%)$, followed by a central venous catheter (CVC; 15.2\%), pus (13.4\%), ascitic fluid $(8.8 \%)$, pleural fluid $(3.0 \%)$, cerebrospinal fluid $(2.8 \%)$, bile $(1.6 \%)$, joint fluid $(1.4 \%)$, tissue $(1.4 \%)$. Other than $C$. metapsilosis and T. asahii, the other 14 species caused bloodstream infections. C. albicans (72/ 224 isolates, $32.1 \%$ ) remained the most common pathogen for bloodstream infections, followed by C. tropicalis (52/ 224 isolates, 23.2\%). The proportion of non-albicans Candida isolates recovered from blood cultures (146/434 isolates, 33.6\%) was significantly higher than that recovered from other specimen types (124/434 isolates, $28.6 \%$ ) $(P<0.5)$. The frequency between blood source and nonblood source isolates was similar for $C$. tropicalis $(12 \%$ versus $13.1 \%$ ) and $C$. parapsilosis complex (8.3\% versus $9 \%$ ). However, there was a significant difference in proportions of blood vs non-blood source for $C$. glabrata $(6.2 \%$ versus $2.7 \%, P<0.05)$ and $C$. pelliculosa $(5.1 \%$ versus $1.6 \%, P<0.01)$. There were also a few uncommon yeast species causing bloodstream infections, such as $R$. mucilaginosa, C. intermedia and K. ohmeri (Table S1).

\section{Concordance Of Initial And Final Identification Results}

The agreement of identification results for yeast isolates obtained from the First Affiliated Hospital of Zhengzhou University and from the central laboratory is presented in Table 2. Overall concordance was 71.2\% (309/434) with the highest observed for $C$. albicans $(97.3 \%, 143 / 147)$, while concordance much lower $(0 \%$ to $76.9 \%)$ for other species. In particular, all isolates of C. metapsilosis, $C$. orthopsilosis, K. ohmeri, R. mucilaginosa, C. intermedia, C. lusitaniae, T. asahii were incorrectly identified or unable to be identified at the First Affiliated Hospital of Zhengzhou University.

Further, accurate identification results to species level by conventional methods from the local hospital showed a relatively high error rate for non- $C$. albicans isolates: $C$. tropicalis $(75 / 109,68.8 \%)$, C. parapsilosis complex sensu stricto (39/69, 56.5\%), C. glabrata (30/39, 76.9\%), C. pelliculosa (8/29, 27.6\%), C. neoformans (8/11, 72.8\%), C. krusei (4/8, 50\%), C. guilliermondii (1/4, 25\%) and $C$. haemulonii $(1 / 4,25 \%)$. Other uncommon minority species were prone to incorrect identification. Only two $R$. mucilaginosa occurred during minor error identification and misidentified to genus level as Rhodotorulaspp. (Table 2).

\section{In Vitro Susceptibilities}

The antifungal susceptibilities of the 434 yeast isolates are presented in Table 3. All three echinocandins exhibited potent in vitro activities against the majority of Candida isolates. All 75 isolates of $C$. parapsilosis complex were susceptible to anidulafungin, micafungin, and caspofungin with $\mathrm{MIC}_{50}$ of 1,1 , and $0.5 \mu \mathrm{g} / \mathrm{mL}$. All the C. albicans were susceptible to micafungin and caspofungin, except one isolate $(0.7 \%)$ showed MIC value to anidulafungin of $0.5 \mu \mathrm{g} / \mathrm{mL}$. Decreased susceptibility to echinocandins was observed among $C$. tropicalis isolates, two isolates displayed resistant to anidulafungin, micafungin, and caspofungin with MICs of 2 and over $8 \mu \mathrm{g} / \mathrm{mL}, 2$ and over $8 \mu \mathrm{g} /$ $\mathrm{mL}$, and 1 and over $8 \mu \mathrm{g} / \mathrm{mL}$. There was one $C$. tropicalis isolate that showed MIC value to anidulafungin and caspofungin of 0.5 and $0.5 \mu \mathrm{g} / \mathrm{mL}$, respectively. Each $C$. glabrata isolate showed intermediate susceptible to anidulafungin or micafungin with MIC of 0.25 or $0.12 \mu \mathrm{g} / \mathrm{mL}$, 
Table 2 Comparison Of Identification Results Of The 434 Isolates Between Those Obtained From The First Affiliated Hospital Of Zhengzhou University (Initial identification) And From The Central Laboratory (Final identification)

\begin{tabular}{|c|c|c|c|c|}
\hline \multirow[t]{3}{*}{ Final Identification } & \multirow{3}{*}{$\begin{array}{l}\text { Number } \\
\text { Of } \\
\text { Isolates }\end{array}$} & \multicolumn{3}{|c|}{ Concordance Of Initial And Final Identification Results } \\
\hline & & \multirow{2}{*}{$\begin{array}{l}\text { Number (\%) Of } \\
\text { Isolates With } \\
\text { Concordance }\end{array}$} & \multicolumn{2}{|c|}{ Number (\%) Of Isolates With Identifcation Errors } \\
\hline & & & $\begin{array}{l}\text { Major } \\
\text { Error }\end{array}$ & Misidentification (Number) \\
\hline Candida albicans & 147 & |43(97.3) & $4(2.7)$ & C. parapsilosis (2), C. tropicalis (I), Stephanoascus ciferrii (I) \\
\hline Candida tropicalis & 109 & $75(68.8)$ & $34(3 \mid .2)$ & $\begin{array}{l}\text { C. albicans (7), C. krusei (7), C. famata (6), C. parapsilosis (5), } \\
\text { C. glabrata (4), C. laurentii (2), C. guilliermondii (I), C. } \\
\text { pelliculosa (I), C. neoformans (I) }\end{array}$ \\
\hline Candida parapsilosis complex & 75 & $39(52)$ & $36(48)$ & \\
\hline Candida parapsilosis sensu stricto & 69 & $39(56.5)$ & $30(43.5)$ & $\begin{array}{l}\text { C. famata (I2), C. laurentii (5), C. albicans (3), C. glabrata (3), } \\
\text { C. krusei (2), C. rugosa (2), C. tropicalis (I), C. pelliculosa (I) }\end{array}$ \\
\hline Candida metapsilosis & 3 & - & $3(100)$ & C. krusei (2), C. laurentii (I) \\
\hline Candida orthopsilosis & 3 & - & $3(100)$ & C. krusei (2), C. famata (I) \\
\hline Candida glabrata & 39 & $30(76.9)$ & $9(23.1)$ & $\begin{array}{l}\text { C.parapsilosis (2), C.tropicalis (2), S.ciferrii (2), C.albicans (I), } \\
\text { C.laurentii (I), Rhodotorula spp. (I) }\end{array}$ \\
\hline Candida pelliculosa & 29 & $8(27.6)$ & $21(72.4)$ & $\begin{array}{l}\text { C. glabrata (I I), C. krusei (5), C. parapsilosis (2), C. albicans } \\
\text { (I), C. norvegensis (I), C. laurentii (I) }\end{array}$ \\
\hline Cryptococcus neoformans & 11 & $8(72.8)$ & $3(27.2)$ & S. ciferrii (I), C. laurentii (I), C. krusei (I) \\
\hline Candida krusei & 8 & $4(50.0)$ & $4(50.0)$ & $\begin{array}{l}\text { C. lipolytica (I), C. glabrata (I), C. pelliculosa (I), C. } \\
\text { neoformans (I) }\end{array}$ \\
\hline Candida guilliermondii & 4 & $\mathrm{I}(25.0)$ & $3(75.0)$ & C. glabrata (2), C. parapsilosis(I) \\
\hline Candida haemulonii & 4 & $\mathrm{I}(25.0)$ & $3(75.0)$ & C. glabrata (2), C. krusei (I) \\
\hline Kodamaea ohmeri & 3 & - & $3(100.0)$ & C. guilliermondii (I), C. pelliculosa (I) C. albicans (I) \\
\hline Rhodotorula mucilaginos $a^{\mathrm{a}}$ & 2 & - & - & - \\
\hline Candida intermedia & 1 & - & $I(100.0)$ & C. glabrata (I) \\
\hline Candida lusitaniae & I & - & $I(100.0)$ & C. laurentii (I) \\
\hline Trichosporon asahii & 1 & - & $I(100.0)$ & C. albicans (I) \\
\hline Total & 434 & $309(71.2)$ & $123(28.3)$ & - \\
\hline
\end{tabular}

Note: a Only two Rhodotorula mucilaginosa isolates occurred during minor error identification and misidentified to genus level as Rhodotorulaspp.

respectively. All echinocandins had MICs of over $8 \mu \mathrm{g} / \mathrm{mL}$ against $C$. neoformans and T. asahii.

Of the azoles, $C$. albicans remained susceptible to all azoles tested during the 3 years (over $95 \%$ susceptible). Of these, voriconazole had a susceptibility rate of $95.9 \%(141 /$ 147 isolates) compared with susceptibility rates of $98 \%$, $99.3 \%$, and $99.3 \%$, for posaconazole, itraconazole, and fluconazole, respectively. Azoles also demonstrated potential in vitro activities against $C$. parapsilosis complex, with susceptibility rate to posaconazole, voriconazole, itraconazole, and fluconazole of $96 \%, 94.7 \%, 100 \%$, and $94.7 \%$, respectively. However, two isolates of C. orthopsilosis exhibited high MICs to posaconazole, voriconazole, and fluconazole $(0.5$, 8 , and $256 \mu \mathrm{g} / \mathrm{mL}$, respectively). There were also one $C$. parapsilosis sensu stricto isolates resistant to posaconazole, voriconazole, and fluconazole with MIC of 0.51 and $16 \mu \mathrm{g} /$ $\mathrm{mL}$, and another $C$. orthopsilosis isolate resistant to voriconazole and fluconazole with MIC of 0.25 and $8 \mu \mathrm{g} / \mathrm{mL}$, respectively. Significant decreased susceptibility was mostly observed in C. tropicalis, and the resistance rate of this 
Table 3 In Vitro Susceptibility Results Of 434 Isolates To Nine Antifungal Agents

\begin{tabular}{|c|c|c|c|c|c|c|}
\hline \multirow[t]{2}{*}{ Species And Agents } & \multicolumn{3}{|c|}{ MIC $(\mu \mathrm{g} / \mathrm{mL})$} & \multicolumn{3}{|c|}{ Number (\%) Of Isolates In Each Category } \\
\hline & Range & $50 \%$ & $90 \%$ & S/WT & I/SDD & R/non-WT \\
\hline \multicolumn{7}{|l|}{ Candida albicans $(n=147)$} \\
\hline Anidulafungin & $\leq 0.015-0.5$ & $\leq 0.015$ & 0.12 & 146(99.3) & $\mathrm{I}(0.7)$ & \\
\hline Micafungin & $\leq 0.008-0.25$ & $\leq 0.008$ & 0.15 & $147(100)$ & & \\
\hline Caspofungin & $0.015-0.25$ & 0.03 & 0.06 & $147(100)$ & & \\
\hline Posaconazole & $\leq 0.08-0.5$ & 0.015 & 0.03 & 144(98) & & $3(2)$ \\
\hline Voriconazole & $\leq 0.08-0.5$ & $\leq 0.08$ & 0.15 & $|4|(95.9)$ & $6(4.1)$ & \\
\hline Itraconazole & $\leq 0.015-0.25$ & 0.03 & 0.06 & 146(99.3) & $\mathrm{I}(0.7)$ & \\
\hline Fluconazole & $\leq 0.12-8$ & 0.25 & 0.5 & I46(99.3) & & $\mathrm{I}(0.7)$ \\
\hline 5-Flucytosine & $\leq 0.06->64$ & $\leq 0.06$ & 0.12 & $145(98.6)$ & & $2(1.4)$ \\
\hline Amphotericin B & $\leq 0.12-2$ & 0.5 & 1 & $147(100)$ & & \\
\hline \multicolumn{7}{|l|}{ Candida tropicalis $(n=109)$} \\
\hline Anidulafungin & $\leq 0.015->8$ & 0.12 & 0.25 & $106(97.2)$ & $\mathrm{I}(0.9)$ & $2(1.8)$ \\
\hline Micafungin & $\leq 0.008->8$ & 0.03 & 0.03 & $107(98.2)$ & & $2(1.8)$ \\
\hline Caspofungin & $0.015->8$ & 0.03 & 0.25 & $106(97.2)$ & $\mathrm{I}(0.9)$ & $2(1.8)$ \\
\hline Posaconazole & $0.015-8$ & 0.25 & 0.6 & $50(45.9)$ & & $56(54.1)$ \\
\hline Voriconazole & $\leq 0.008->8$ & 0.12 & 0.5 & $67(61.5)$ & $32(29.4)$ & $10(9.1)$ \\
\hline Itraconazole & $0.03->16$ & 0.25 & 0.5 & $100(91.7)$ & & $9(8.3)$ \\
\hline Fluconazole & $0.25->256$ & 2 & 16 & $86(78.9)$ & $10(9.2)$ & $13(11.9)$ \\
\hline 5-Flucytosine & $\leq 0.06-64$ & $\leq 0.06$ & 0.12 & $107(98.2)$ & & $2(1.8)$ \\
\hline Amphotericin B & $\leq 0.12-4$ & 1 & I & 107(98.2) & & $2(1.8)$ \\
\hline \multicolumn{7}{|c|}{ Candida parapsilosis complex $(\mathrm{n}=75)^{\mathrm{a}}$} \\
\hline Anidulafungin & $0.03-2$ & I & 2 & $75(100)$ & & \\
\hline Micafungin & $0.06-2$ & I & 1 & $75(100)$ & & \\
\hline Caspofungin & $0.06-1$ & 0.5 & 0.5 & $75(100)$ & & \\
\hline Posaconazole & $\leq 0.008-0.5$ & 0.03 & 0.06 & $72(96)$ & & $3(4)$ \\
\hline Voriconazole & $\leq 0.008-8$ & $\leq 0.008$ & 0.03 & $7 I(94.7)$ & & $4(5.3)$ \\
\hline Itraconazole & $\leq 0.015-0.5$ & 0.03 & 0.12 & $75(100)$ & & \\
\hline Fluconazole & $\leq 0.12-256$ & 0.5 & 2 & $71(94.7)$ & & $4(5.3)$ \\
\hline 5-Flucytosine & $\leq 0.06->64$ & $\leq 0.06$ & 0.25 & $70(93.3)$ & & $5(6.7)$ \\
\hline Amphotericin B & $0.25-2$ & 0.5 & 1 & $75(100)$ & & \\
\hline \multicolumn{7}{|l|}{ Candida glabrata $(\mathrm{n}=39)$} \\
\hline Anidulafungin & $\leq 0.015-0.25$ & 0.03 & 0.12 & $38(97.4)$ & $\mathrm{I}(2.6)$ & \\
\hline Micafungin & $\leq 0.008-0.12$ & 0.015 & 0.03 & $38(97.4)$ & $I(2.6)$ & \\
\hline Caspofungin & $0.03-0.12$ & 0.06 & 0.12 & $39(100)$ & & \\
\hline Posaconazole & $0.03->8$ & 0.5 & 2 & $35(89.7)$ & & $4(10.3)$ \\
\hline Voriconazole & $0.015->8$ & 0.25 & 1 & $28(71.8)$ & & $\mathrm{II}(28.2)$ \\
\hline Itraconazole & $0.03->16$ & 0.5 & 1 & $36(92.3)$ & & $3(7.7)$ \\
\hline Fluconazole & $0.5->256$ & 8 & 32 & $36(92.3)$ & & $3(7.7)$ \\
\hline 5-Flucytosine & $\leq 0.06->64$ & $\leq 0.06$ & $\leq 0.06$ & $38(97.4)$ & & $\mathrm{I}(2.6)$ \\
\hline Amphotericin B & $0.25-4$ & I & I & $38(97.4)$ & & $\mathrm{I}(2.6)$ \\
\hline \multicolumn{7}{|l|}{ Candida pelliculosa $(\mathrm{n}=29)$} \\
\hline Anidulafungin & $\leq 0.015-0.06$ & $\leq 0.015$ & $\leq 0.015$ & & & \\
\hline Micafungin & $0.03-0.06$ & 0.03 & 0.06 & & & \\
\hline Caspofungin & $\leq 0.008-0.12$ & 0.06 & 0.06 & $29(100)$ & & \\
\hline Posaconazole & $0.12->8$ & 0.5 & 2 & $28(96.6)$ & & $\mathrm{I}(3.4)$ \\
\hline
\end{tabular}

(Continued) 
Table 3 (Continued).

\begin{tabular}{|c|c|c|c|c|c|c|}
\hline \multirow[t]{2}{*}{ Species And Agents } & \multicolumn{3}{|c|}{$\operatorname{MIC}(\mu \mathrm{g} / \mathrm{mL})$} & \multicolumn{3}{|c|}{ Number (\%) Of Isolates In Each Category } \\
\hline & Range & $50 \%$ & $90 \%$ & S/WT & I/SDD & R/non-WT \\
\hline Voriconazole & $0.06->8$ & 0.25 & 0.5 & $17(58.6)$ & & $\mid 2(4 \mid .4)$ \\
\hline Itraconazole & $0.06->16$ & 0.25 & 0.5 & & & \\
\hline Fluconazole & $2->256$ & 8 & 16 & $13(44.5)$ & & $16(55.2)$ \\
\hline 5-Flucytosine & $\leq 0.06->64$ & 2 & 32 & & & \\
\hline Amphotericin B & $0.25-1$ & I & 1 & & & \\
\hline \multicolumn{7}{|c|}{ Cryptococcus neoformans $(\mathrm{n}=\mathrm{II})$} \\
\hline Anidulafungin & $>8$ & $>8$ & $>8$ & & & \\
\hline Micafungin & $>8$ & $>8$ & $>8$ & & & \\
\hline Caspofungin & $>8$ & $>8$ & $>8$ & & & \\
\hline Posaconazole & $0.06-0.25$ & 0.12 & 0.12 & & & \\
\hline Voriconazole & $0.03-0.06$ & 0.03 & 0.06 & & & \\
\hline Itraconazole & $0.03-0.12$ & 0.06 & 0.12 & & & \\
\hline Fluconazole & $2-8$ & 4 & 4 & & & \\
\hline 5-Flucytosine & $0.5-4$ & 2 & 4 & & & \\
\hline Amphotericin B & $0.5-1$ & 0.5 & 1 & & & \\
\hline \multicolumn{7}{|l|}{ Other species $(n=24)^{b}$} \\
\hline Anidulafungin & $\leq 0.015->8$ & I & 2 & & & \\
\hline Micafungin & $0.015->8$ & 0.12 & 0.5 & & & \\
\hline Caspofungin & $0.03->8$ & 0.12 & 2 & & & \\
\hline Posaconazole & $0.015-1$ & 0.25 & 1 & & & \\
\hline Voriconazole & $\leq 0.008->8$ & 0.25 & $>8$ & & & \\
\hline Itraconazole & $0.03->16$ & 0.25 & I & & & \\
\hline Fluconazole & $0.5->256$ & 16 & $>256$ & & & \\
\hline 5-Flucytosine & $\leq 0.06->64$ & 8 & 64 & & & \\
\hline Amphotericin B & $\leq 0.12-4$ & 0.25 & 4 & & & \\
\hline
\end{tabular}

Note: ${ }^{a}$ This includes Candida parapsilosis sensu stricto (69 isolates), Candida metapsilosis (3 isolates), Candida orthopsilosis ( 3 isolates). ${ }^{\mathrm{b}}$ This included Candida krusei $(8$ isolates), Candida guilliermondii (4 isolates), Candida haemulonii (4 isolates), Candida lusitaniae (I isolate), Candida intermedia (I isolate), Kodamaea ohmeri (3 isolates), Rhodotorula mucilaginosa ( 2 isolates) and Trichosporon asahii (I isolate).

Abbreviations: S, Susceptible; SDD, Susceptible-dose dependent; I, Intermediate; WT, Wild-type; R, Resistant.

species to posaconazole, voriconazole, itraconazole, and fluconazole of $54.1 \%, 9.1 \%, 8.3 \%$, and $11.9 \%$, respectively. In addition, the non-susceptibility of $C$. tropicalis to both fluconazole and voriconazole clearly increased to $27.5 \%$ and $44.8 \%$ in 2014 , distinctly higher than those of $14.7 \%$ and $32.4 \%$ in 2012 , but no statistical significance $(P<0.5)$ was shown. Similarly, a decreased susceptibility trend was observed among the C. glabrata isolates, especially for the overall voriconazole resistance rate already up to $28.2 \%$, followed by the resistance rate to posaconazole, itraconazole, and fluconazole of $10.3 \%, 7.7 \%$, and $7.7 \%$, respectively. $C$. pelliculosa exhibited extremely high rates of resistance to fluconazole and voriconazole that reach up to $55.2 \%$ and $41.4 \%$, respectively (Table 3 ).

Azoles showed a good in vitro antifungal activity against $C$. neoformans to posaconazole, voriconazole, itraconazole, and fluconazole with the $\mathrm{MIC}_{90}$ of 0.12 , $0.06,0.12$, and $4 \mu \mathrm{g} / \mathrm{mL}$, respectively. Other uncommon yeast species displayed generally high MIC values to four azole agents. It is noteworthy that three of four C. haemulonii isolates showed high MIC values to posaconazole, voriconazole, itraconazole, and fluconazole of $1,>8,>16$, and $>256 \mu \mathrm{g} / \mathrm{mL}$, respectively.

All 147 C. albicans isolates showed a WT phenotype to amphotericin B while two isolates of this species $(1.4 \%)$ were non-WT phenotype to 5-flucytosine. And, $2.6 \%$ of $C$. glabrata (one isolate) and $1.8 \%$ of $C$. tropicalis (two isolates) had non-WT phenotype both to amphotericin B and 5-flucytosine. All isolates of $C$. parapsilosis complex were susceptible to amphotericin B, but $6.7 \%$ of this species (5 isolates) were resistant to 5-flucytosine. $C$. pelliculosa and other yeast species possessed higher 
$\mathrm{MIC}_{50}$ to flucytosine of 2 and $8 \mu \mathrm{g} / \mathrm{mL}$, respectiveluy. The $\mathrm{MIC}_{50}$ value of amphotericin B for all species tested were 0.25 to $1 \mu \mathrm{g} / \mathrm{mL}$.

\section{Discussion}

This retrospective observational study describes a laboratory-based, 3-year surveillance of invasive yeast infections in the First Affiliated Hospital of Zhengzhou University. Although national data have been published, local data of species distribution, patient characteristics, and antifungal susceptibility profiles are essential to inform early and effective treatment for Candida infections.

Although there are many large-scale, global surveillance programs identifying species distribution and resistance trends of IFIs, ${ }^{5,9,15}$ similar studies in medical institutions within China are few. ${ }^{16-19}$ Invasive candidiasis remains the most common invasive yeast infection, accounting for $96.1 \%$ of the episodes in this study. Generally, although C. albicans remains the dominant species, it accounted for only $33.9 \%$ of the isolates collected in this study, lower than that overall in the Asia-Pacific region (46.3\%), ${ }^{9}$ another Chinese study (43$47.4 \%)^{5}$ and more specifically, in Beijing $(50.3 \%)^{10}$ during the same period. C. tropicalis was the most common nonalbicans Candida species (25.1\%), which again differs from the epidemiology reported in the Asia-Pacific region of SENTRY antimicrobial surveillance program, where $C$. glabrata was second most common species $(19.3 \%)^{9}$ and contrasting with the CHIF-NET Study in China. ${ }^{5}$ However, the relative frequency of $C$. tropicalis in our study was similar to other single-center studies. ${ }^{16,18}$ Of interest, we observed a relatively high frequency of $C$. pelliculosa $(6.7 \%)$ isolates at our hospital (similar to the frequency of C. glabrata, 9\%) with the majority of isolates being from blood (81.5\%). The above findings emphasize the necessity to perform locally relevant epidemiological studies.

From the clinical perspective, the frequency of IFIs in the patients older than 65 years deserves attention in keeping with previous observations that infection with some common Candida species, such as $C$. albicans, $C$. parapsilosis, C. tropicalis, and C. glabrata, may be associated with older age. ${ }^{10,16,20}$ Conversely, the isolation of $C$. pelliculosa in our study was associated with disease in newborns (35.5\%). There have been reports of nosocomial transmission of $C$. pelliculosa fungemia in the neonatal ICU, ${ }^{21,22}$ and clinicians should be vigilant about the potential presence of this species. Our study also confirmed that patients admitted to ICUs and surgical department pose a high risk of developing IFIs. ${ }^{17,23}$ MALDI-TOF MS has been extensively proven as a powerful tool in the identification of yeast species compared with traditional identification methods. $^{12,26}$ To this end, incorrect identification results in our study using the CHROMagar Candida medium and the VITEK $2 \AA$ compact system not only occurred for uncommon Candida isolates but also for common Candida species (error rates from 2.7\% of C. albicans up to $48 \%$ of the $C$. parapsilosis complex). For the common Candida species, conventional identification methods especially exhibited poor identification performance to the $C$. parapsilosis complex with $48 \%$ of the error identification rate. Of these, C. parapsilosis sensu stricto were most indistinguishable from $C$. famata and $C$. laurentii while $C$. metapsilosis and $C$. orthopsilosis were liable to misidentified as C. krusei. Some studies have highlighted the inferior identification performance of common commercial systems to $C$. parapsilosis complex, and C. famata has also been reported as the most primary species misidentified from $C$. parapsilosis sensu stricto. ${ }^{27,28}$ As is well known, the performance of conventional identification methods to less commonly encountered yeast species is unsatisfactory. ${ }^{29,30}$ We observed high misidentification rates in the identification of $C$. pelliculosa (72.4\%), $C$. krusei (50\%), C. guilliermondii (75\%), C. haemulonii $(75 \%)$, and $K$. ohmeri (100\%). In consideration of molecular techniques associated with increased costs, longer turn-around time and the need for considerable expertise, ${ }^{29}$ Zhang et al proposed an algorithm of MALDI-TOF MS supplied with DNA sequencing for yeast identification that can be applied to epidemiological investigation and the routine laboratory identification. ${ }^{12}$

C. albicans exhibited low antifungal resistance rates as has been shown globally. $5,9,10$ The majority of the $C$. parapsilosis complex isolates were susceptible (or WT) to all nine antifungals tested ( $\geq 93.3 \%$ ), with a $5.3 \%$ of resistance rate to fluconazole and voriconazole. The findings were in general higher than those obtained from the national CHIF-NET surveillance. $^{5,31}$ Among the $C$. parapsilosis complex, $C$. orthopsilosis was the most resistant species and had very high MICs to azoles ( 8 or $256 \mu \mathrm{g} / \mathrm{mL}$ for fluconazole). Although $C$. orthopsilosis is relatively uncommon, it is essential to perform accurate identification and antifungal susceptibility test to detect such species. ${ }^{32}$ High-level azole resistance was mainly observed in $C$. tropicalis, with resistance rates to fluconazole of $11.9 \%$, and to voriconazole of $9.1 \%$, both higher than that seen in the SENTRY Antifungal Surveillance Program (1997-2016) (9.2\% fluconazole-resistance), ${ }^{9}$ national level (2010-2012) (both of 5.7\%), ${ }^{31}$ and a teaching hospital in 
southwest China $(2.2 \%$ and $0 \%),{ }^{16}$ but similar to that in the national level during a 5-year surveillance $(12.8 \%$ and $11.4 \%)^{33}$ and Beijing hospitals (both of $9.4 \%$ ). ${ }^{10}$ Fan et al have underlined the notable increasing trend in azole nonsusceptible invasive $C$. tropicalis infection in China. Our research data accorded with this epidemical tendency with an even higher voriconazole non-susceptible rate. ${ }^{33}$ Thus, continuous surveillance, molecular epidemiology, and resistance mechanism study are essential, and empirical therapeutic strategies for C. tropicalis invasive infections may be modified in China. ${ }^{33,34}$ Moreover, in vitro susceptibility results showed that only $7.7 \%$ of C. glabrata isolates were resistant or nonWT to all four azoles similar to results reported in a 5-year multicenter study based on CHIF-NET program ${ }^{35}$ but contrast with results in the USA. ${ }^{36}$ Although a rising echinocandin resistance of $C$. glabrata in the USA poses a serious challenge for clinical therapy, ${ }^{37}$ the fact that only one isolate $(2.6 \%)$ herein was considered to be of "intermediate susceptibility" to anidulafungin and micafungin is reassuring. Interestingly, our results showed that a high proportion of C. pelliculosa isolates $-55.2 \%$ and $41.4 \%$ were non-WT to fluconazole and voriconazole, respectively. Such high resistance rates have not been previously reported ${ }^{11,38}$. Other yeast species, although uncommon, exhibited high MIC values to the four azole agents. For example, C. haemulonii was highly resistant to the azoles, amphotericin B, and 5-flucytosine but had low MICs for the echinocandins in our study. This resistance pattern is similar to that reported by Hou et al in China and Ramos et al in Brazil. ${ }^{39,40}$

Two main limitations of this study are mentioned. First, we used the YO10 methodology to perform antifungal susceptibility testing and not a reference broth microdilution method. However, the essential agreement between this methodology and the CLSI and the EUCAST reference procedures had been proven to be very high for yeast species. $^{35,41,42}$ Importantly, surveillance work was interrupted for a number of reasons beyond our control from 2014 to 2016 limiting the collection of more complete epidemiological data.

In conclusion, the present study provides valuable local surveillance data on the epidemiology and antifungal susceptibilities of invasive yeast species isolated from the First Affiliated Hospital of Zhengzhou University, which can be used to guide the selection of both empiric and targeted antifungal therapy. Although C. albicans remained the most prevalent species, the frequency of $C$. tropicalis and its notable and increasing trend azole non-susceptibility requires noting. Less common Candida species also exhibited high azole resistance rates, continuously monitoring of local epidemiology of invasive yeast infection and of antifungal resistance is warranted.

\section{Acknowledgments}

This work was supported by the National Nature Science Foundation of China ((81802049, 81572057), the Innovation Fund of Peking Union Medical College (No. 2018-1002-0102), Henan National Innovation Demonstration Zone Leading Biopharmaceutical Industry Cluster Project (grant no. 181200211900), the Fundamental Research Funds for the Central Universities(3332018035), and Beijing Natural Science Foundation (5184037).

\section{Author Contributions}

All authors contributed to data analysis, drafting or revising the article, gave final approval of the version to be published, and agree to be accountable for all aspects of the work.

\section{Disclosure}

The authors report no further conflicts of interest in this work.

\section{References}

1. Miceli MH, Diaz JA, Lee SA. Emerging opportunistic yeast infections. Lancet Infect Dis. 2011;11(2):142-151. doi:10.1016/S14733099(10)70218-8

2. Pfaller MA, Diekema DJ. Epidemiology of invasive candidiasis: a persistent public health problem. Clin Microbiol Rev. 2007;20 (1):133-163. doi:10.1128/CMR.00029-06

3. Xiao M, Chen SCA, Kong FR, et al. Five-year China Hospital Invasive Fungal Surveillance Net (CHIF-NET) study of invasive fungal infections caused by noncandidal yeasts: species distribution and azole susceptibility. Infect Drug Resist. 2018;11:1659-1667.

4. Toth R, Nosek J, Mora-Montes HM, et al. Candida parapsilosis: from genes to the bedside. Clin Microbiol Rev. 2019;32:2.

5. Xiao M, Sun ZY, Kang M, et al. Five-year national surveillance of invasive candidiasis: species distribution and azole susceptibility from the China Hospital Invasive Fungal Surveillance Net (CHIFNET) study. J Clin Microbiol. 2018;56:7. doi:10.1128/JCM.00577-18

6. Perlin DS, Rautemaa-Richardson R, Alastruey-Izquierdo A. The global problem of antifungal resistance: prevalence, mechanisms, and management. Lancet Infect Dis. 2017;17(12):E383-E392. doi:10.1016/ S1473-3099(17)30316-X

7. Grim SA, Berger K, Teng C, et al. Timing of susceptibility-based antifungal drug administration in patients with Candida bloodstream infection: correlation with outcomes. J Antimicrob Chemoth. 2012;67 (3):707-714. doi:10.1093/jac/dkr511

8. McCarty TP, Pappas PG. Invasive Candidiasis. Infect Dis Clin North Am. 2016;30(1):103-124. doi:10.1016/j.idc.2015.10.013

9. Pfaller MA, Diekema DJ, Turnidge JD, Castanheira M, Jones RN. Twenty years of the SENTRY antifungal surveillance program: results for Candida species from 1997-2016. Open Forum Infect Dis. 2019;6(Suppl 1):S79-S94. doi:10.1093/ofid/ofy358

10. Guo LN, Xiao M, Cao B, et al. Epidemiology and antifungal susceptibilities of yeast isolates causing invasive infections across urban Beijing, China. Future Microbiol. 2017;12(12):1075-1086. 
11. Wang $\mathrm{H}$, Xiao M, Chen SCA, et al. In vitro susceptibilities of yeast species to fluconazole and voriconazole as determined by the 2010 National China Hospital Invasive Fungal Surveillance Net (CHIF-NET) study. J Clin Microbiol. 2012;50(12):3952-3959. doi:10.1128/JCM.01130-12

12. Zhang L, Xiao M, Wang H, et al. Yeast identification algorithm based on use of the vitek MS system selectively supplemented with ribosomal DNA sequencing: proposal of a reference assay for invasive fungal surveillance programs in China. J Clin Microbiol. 2014;52 (2):572-577. doi:10.1128/JCM.02543-13

13. Clinical and Laboratory Standards Institute. Performance Standards for Antifungal Susceptibility Testing of Yeasts. 1st ed. Wayne, PA: Clinical and Laboratory Standards Institute; 2017.

14. Clinical and Laboratory Standards Institute. Epidemiological Cutoff Values for Antifungal Susceptibility Testing. 2nd ed. Wayne, PA: Clinical and Laboratory Standards Institute; 2018.

15. Pfaller MA, Diekema DJ. Progress in antifungal susceptibility testing of Candida spp. by use of clinical and laboratory standards institute broth microdilution methods, 2010 to 2012. J Clin Microbiol. 2012;50(9):2846-2856. doi:10.1128/JCM.00937-12

16. Pu SL, Niu SQ, Zhang CM, et al. Epidemiology, antifungal susceptibilities, and risk factors for invasive candidiasis from 2011 to 2013 in a teaching hospital in southwest China. J Microbiol Immunol. 2017;50(1):97-103.

17. Jia XJ, Li CY, Cao J, Wu XN, Zhang LP. Clinical characteristics and predictors of mortality in patients with candidemia: a six-year retrospective study. Eur J Clin Microbiol. 2018;37(9):1717-1724. doi:10.1007/s10096-018-3304-9

18. $\mathrm{Li} \mathrm{W}, \mathrm{Hu} \mathrm{YA,} \mathrm{Li} \mathrm{FQ,} \mathrm{et} \mathrm{al.} \mathrm{Distribution} \mathrm{of} \mathrm{yeast} \mathrm{isolates} \mathrm{from} \mathrm{invasive}$ infections and their in vitro susceptibility to antifungal agents: evidence from 299 cases in a 3-Year (2010 to 2012) surveillance study. Mycopathologia. 2015;179(5-6):397-405. doi:10.1007/s11046-0159858-5

19. Wang SH, Shen M, Lin HC, Sun PL, Lo HJ, Lu JJ. Molecular epidemiology of invasive Candida albicans at a tertiary hospital in northern Taiwan from 2003 to 2011. Med Mycol. 2015;53(8):828836. doi:10.1093/mmy/myv065

20. Yang ZT, Wu L, Liu XY, et al. Epidemiology, species distribution and outcome of nosocomial Candida spp. bloodstream infection in Shanghai. BMC Infect Dis. 2014;14:241. doi:10.1186/1471-2334-14-241

21. da Silva CM, Parahym AMRD, Leao MPC, de Oliveira NT, Amorim RDM, Neves RP. Fungemia by Candida pelliculosa (Pichia anomala) in a neonatal intensive care unit: a possible clonal origin. Mycopathologia. 2013;175(1-2):175-179. doi:10.1007/s11046-012-9605-0

22. Lin HC, Lin HY, Su BH, et al. Reporting an outbreak of Candida pelliculosa fungemia in a neonatal intensive care unit. $J$ Microbiol Immunol. 2013;46(6):456-462.

23. Jose $\mathrm{P}$, Alvarez-Lerma F, Maseda $\mathrm{E}$, et al. Invasive fungal infection in critically ill patients: hurdles and next challenges. $J$ Chemotherapy. 2019;31(2):64-73. doi:10.1080/1120009X.2018.1557799

24. Soldini S, Posteraro B, Vella A, et al. Microbiologic and clinical characteristics of biofilm-forming Candida parapsilosis isolates associated with fungaemia and their impact on mortality. Clin Microbiol Infec. 2018;24(7):771-777. doi:10.1016/j.cmi.2017.11.005

25. Silva-Dias A, Miranda IM, Branco J, Monteiro-Soares M, Pina-Vaz C, Rodrigues AG. Adhesion, biofilm formation, cell surface hydrophobicity, and antifungal planktonic susceptibility: relationship among Candida spp. Front Microbiol. 2015;6:205. doi:10.3389/fmicb.2015.00205

26. Dhiman N, Hall L, Wohlfiel SL, Buckwalter SP, Wengenack NL. Performance and cost analysis of matrix-assisted laser desorption ionization-time of flight mass spectrometry for routine identification of yeast. $J$ Clin Microbiol. 2011;49(4):1614-1616. doi:10.1128/JCM.02381-10

27. Karabicak N, Uludag Altun H, Karatuna O, et al. [Evaluation of common commercial systems for the identification of yeast isolates in microbiology laboratories: a multicenter study]. Mikrobiyol Bul. 2015;49(2):210-220. doi:10.5578/mb.9370
28. Castanheira M, Woosley LN, Diekema DJ, Jones RN, Pfaller MA. Candida guilliermondii and other species of candida misidentified as Candida famata: assessment by Vitek 2, DNA sequencing analysis, and matrix-assisted laser desorption ionization-time of flight mass spectrometry in two global antifungal surveillance programs. Mikrobiyol Bul. 2013;51(1):117-124.

29. Chao QT, Lee TF, Teng SH, et al. Comparison of the accuracy of two conventional phenotypic methods and two MALDI-TOF MS systems with that of DNA sequencing analysis for correctly identifying clinically encountered yeasts. PLoS ONE. 2014;9(10):e109376. doi:10.1371/journal.pone. 0109376

30. Zhao Y, Tsang CC, Xiao M, et al. Yeast identification by sequencing, biochemical kits, MALDI-TOF MS and rep-PCR DNA fingerprinting. Med Mycol. 2018;56(7):816-827. doi:10.1093/mmy/ myx 118

31. Xiao M, Fan X, Chen SCA, et al. Antifungal susceptibilities of Candida glabrata species complex, Candida krusei, Candida parapsilosis species complex and Candida tropicalis causing invasive candidiasis in China: 3 year national surveillance. $J$ Antimicrob Chemoth. 2015;70(3):802-810. doi:10.1093/jac/dku460

32. Canton E, Peman J, Quindos G, et al. Prospective multicenter study of the epidemiology, molecular identification, and antifungal susceptibility of Candida parapsilosis, Candida orthopsilosis, and Candida metapsilosis isolated from patients with candidemia. Antimicrob Agents Chemother. 2011;55(12):5590-5596. doi:10.1128/AAC.00466-11

33. Fan X, Xiao M, Liao K, et al. Notable increasing trend in azole nonsusceptible Candida tropicalis causing invasive candidiasis in China (August 2009 to July 2014): molecular epidemiology and clinical azole consumption. Front Microbiol. 2017;8:464. doi:10.3389/fmicb. 2017.00464

34. Fan X, Xiao M, Zhang D, et al. Molecular mechanisms of azole resistance in Candida tropicalis isolates causing invasive candidiasis in China. Clin Microbiol Infect. 2018.

35. Hou X, Xiao M, Chen SCA, et al. Molecular epidemiology and antifungal susceptibility of Candida glabrata in China (August 2009 to July 2014): a multi-center study. Front Microbiol. 2017;8:880. doi:10.3389/fmicb.2017.00880

36. Pfaller MA, Castanheira M, Lockhart SR, Ahlquist AM, Messer SA, Jones RN. Frequency of decreased susceptibility and resistance to echinocandins among fluconazole-resistant bloodstream isolates of Candida glabrata. J Clin Microbiol. 2012;50(4):1199-1203. doi:10.1128/JCM.06112-11

37. Perlin DS, Shor E, Zhao Y. Update on antifungal drug resistance. Curr Clin Microbiol Rep. 2015;2(2):84-95. doi:10.1007/s40588-0150015-1

38. Suhr MJ, Gomes-Neto JC, Banjara N, et al. Epidemiological investigation of Candida species causing bloodstream infection in paediatric small bowel transplant recipients. Mycoses. 2017;60(6):366-374. doi:10.1111/myc.2017.60.issue-6

39. Hou X, Xiao M, Chen SCA, et al. Identification and antifungal susceptibility profiles of Candida haemulonii species complex clinical isolates from a multicenter study in China. $J$ Clin Microbiol. 2016;54(11):2676-2680. doi:10.1128/JCM.01492-16

40. Ramos LS, Figueiredo-Carvalho MHG, Barbedo LS, et al. Candida haemulonii complex: species identification and antifungal susceptibility profiles of clinical isolates from Brazil. J Antimicrob Chemoth. 2015;70(1):111-115. doi:10.1093/jac/dku321

41. Espinel-Ingroff A, Pfaller M, Messer SA, Knapp CC, Holliday N, Killian SB. Multicenter comparison of the Sensititre YeastOne colorimetric antifungal panel with the NCCLS M27-A2 reference method for testing new antifungal agents against clinical isolates of Candida spp. J Clin Microbiol. 2004;42(2):718-721. doi:10.1128/ JCM.42.2.718-721.2004

42. Posteraro B, Sanguinetti M. The future of fungal susceptibility testing. Future Microbiol. 2014;9(8):947-967. doi:10.2217/fmb.14.55 


\section{Publish your work in this journal}

Infection and Drug Resistance is an international, peer-reviewed openaccess journal that focuses on the optimal treatment of infection (bacterial, fungal and viral) and the development and institution of preventive strategies to minimize the development and spread of resistance. The journal is specifically concerned with the epidemiology of

antibiotic resistance and the mechanisms of resistance development and diffusion in both hospitals and the community. The manuscript management system is completely online and includes a very quick and fair peerreview system, which is all easy to use. Visit http://www.dovepress.com/ testimonials.php to read real quotes from published authors. 